\title{
Shaping the Way from the Unknown to the Known: The Role of Convex Hull Shape in Numerical Comparisons
}

\author{
Yoel Shilat ${ }^{1,2}$ iD , Moti Salti2,3 (D), Avishai Henik ${ }^{1,2}$ (D)
}

${ }^{1}$ Department of Psychology, Ben-Gurion University of the Negev, Israel

${ }^{2}$ Zlotowski Center for Neuroscience, Ben-Gurion University of the Negev, Israel

${ }^{3}$ Brain Imaging Research Center, Ben-Gurion University of the Negev, Israel

Correspondence:

yoel.shilat@gmail.com 


\begin{abstract}
Various studies have shown that numerical processing is modulated by non-numerical physical properties. One such physical property is the convex hull - the smallest convex polygon surrounding all items in an array. The convex hull is usually discussed only in terms of its area. However, our group has shown that observers use the convex hull shape, as defined according to the number of vertices of the convex hull, to make numerical estimations (Katzin et al., 2020). Yet, it is still unknown if and how the convex hull shape affects comparison tasks, and how it interacts with its counterpart, convex hull area. Here we re-examine the data collected by Katzin and colleagues (2019). Using image processing, we extracted the information on the convex hull shape and showed that the shape affects latency and accuracy of numerical comparisons. We found that both the convex hull shape and other physical properties (i.e., convex hull area, average diameter, density, total circumference, and total surface area) have distinct effects on performance. Finally, the convex hull shape effect was found in counting and estimation ranges, however its effect decreased with numerosities above the counting range. Our results indicate that the interplay between convex hull shape and other physical properties, including convex hull area and numerosity, plays an important role in numerical decisions. We suggest that the convex hull shape should be controlled for when designing non-symbolic numerical tasks.
\end{abstract}

Keywords: numerical cognition, physical properties, non-symbolic arrays, convex hull, shape 


\section{Shaping the Way from the Unknown to the Known: The Role of Convex Hull Shape in Numerical Comparisons}

\section{Introduction}

Cognitive research strives to examine the interplay between low-level (perceptual) properties and high-level (conceptual) properties. This interplay might modulate even highlevel conceptual decisions. Recent research in numerical cognition is an excellent ground to study this issue. The current study examines how certain perceptual properties affect frequent daily actions - the comparative judgments of numerosities.

\subsection{Numerosity and physical properties}

Numerical processing is not reserved for mathematicians only; we constantly process the number of elements in our surrounding. Numerical comparisons between non-symbolic arrays is a daily task, such as when we want to choose the less numerous (shorter) line in the supermarket (Leibovich, Katzin, Harel, \& Henik, 2017). An array's numerosity is inseparable from its physical properties as more dots frequently encompass more area, will be denser, and so on. Various studies have shown that people rely on physical properties in order to process numerosity of non-symbolic arrays (Gebuis \& Reynvoet, 2011; Leibovich et al., 2017; Soltész, Szűcs, \& Szűcs, 2010). Importantly, different physical properties are correlated with numerosity and are intercorrelated with one another (Salti, Katzin, Katzin, Leibovich, \& Henik, 2017). Two arrays with different numerosities cannot have the same physical properties (Katzin, Salti, \& Henik, 2019; Leibovich \& Henik, 2013). Therefore, it is impossible to dissociate the physical properties and numerosity of non-symbolic arrays (Gebuis \& Reynvoet, 2011; Leibovich et al., 2017; Salti et al., 2017).

Throughout the years, numerous studies have used Stroop-like non-symbolic numerical comparison tasks to test the role of different physical properties in numerical processing (Gebuis \& Reynvoet, 2012; Henik \& Tzelgov, 1982). In a numerical comparison task, two arrays are displayed and the participants have to choose the more numerous one. Manipulating the arrays' physical properties can change their correlation with numerosity. 
Consequently, the amount of information provided by physical properties on numerosity changes. A common method to control the amount of information provided by physical properties is the use of congruency (Egner, 2007; Henik \& Tzelgov, 1982). Two stimuli are congruent when two of their features display a high degree of perceptual, conceptual, or structural similarity (Egner, 2007). A non-symbolic numerical stimulus of two arrays is congruent when the more numerous array is also larger in its physical properties. In contrast, a stimulus is incongruent when the less numerous array has larger physical properties (Gebuis et al., 2009; Leibovich et al., 2017). For example, in a congruent stimulus, the total surface area of the more numerous array is larger than the total surface area of the less numerous array (Gebuis, Cohen Kadosh, de Haan, \& Henik, 2009). This convergence of numerosity and the physical domain facilitates responding (Nys \& Content, 2011; Rousselle, Palmers, \& Noël, 2004). In an incongruent trial, the less numerous array has a larger total surface area. This mismatch between numerosity and physical properties results in an interference to the response (Nys \& Content, 2011; Rousselle et al., 2004). The degree of interference is quantified using the congruency effect (based on reaction time [RT], i.e., RTincongruent - RTcongruent). A bigger congruency effect of a certain physical property indicates that it has a bigger role in numerical processing.

Salti el al. (2017) put forward a taxonomy of the physical properties that are intercorrelated with numerosity into intrinsic and extrinsic properties (see Figure 1). This taxonomy allows predicting the role of the different physical properties according to the amount of information they convey. Although intimately correlated, intrinsic and extrinsic properties provide different types of information. (DeWind, Adams, Platt, \& Brannon, 2015; Gebuis \& Reynvoet, 2011; Piazza, Izard, Pinel, Bihan, \& Dehaene, 2004; Salti et al., 2017). While extrinsic properties provide data on the whole array, intrinsic properties do not. Intrinsic properties, such as diameter, circumference or surface area, are based on a calculation that uses information on each individual dot. Therefore, although extrinsic and intrinsic properties have different correlations among them, they provide different amounts of information, such that extrinsic representation is more informative and efficient regarding numerosity than intrinsic representation. 


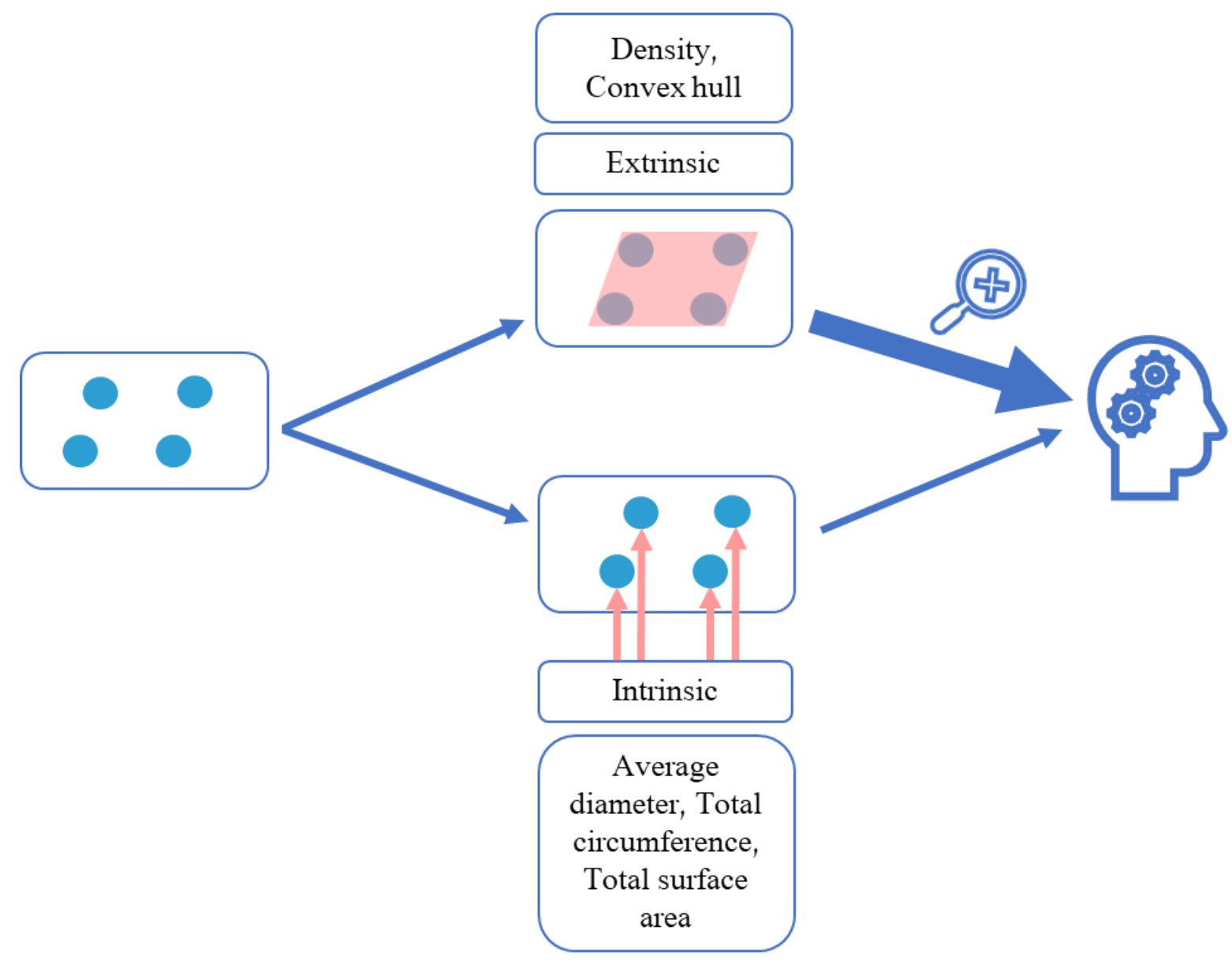

Fig. 1. A taxonomy of the physical properties correlated with numerosity. The properties of the array can be classified into two types: intrinsic and extrinsic properties. Intrinsic properties can be calculated on each individual dot. Extrinsic properties can only be calculated on an array of dots and are more informative than intrinsic properties. Out of all of the extrinsic properties, the convex hull (see the pink parallelogram surrounding the top array) provides a more efficient representation than density since it is able to represent the whole array without encoding information for every single item.

Extrinsic properties include density and the convex hull $(\mathrm{CH})$ and consider the locations and sizes of the elements. The $\mathrm{CH}$ is the smallest polygon containing all objects in an array with all of its angles less than or equal to 180 degrees (Katzin et al., 2019; Weisstein, 2002). A common way to describe the $\mathrm{CH}$ is the shape that emerges if a rubber band is wrapped around the array (Bentley, Kung, Schkolnick, \& Thompson, 1978; Day, 1988; Liao, Lu, Ma, $\mathrm{Xue}, \& \mathrm{Yu}, 2012)$. The $\mathrm{CH}$ has two main distinct properties: its area and its shape (Cabo \& Groeneboom, 1994; Efron, 1965) and both are extrinsic properties. The convex hull area $(\mathrm{CH}-$ 
Area) is defined according to the smallest contour containing all objects in the array and spanning between the objects that create this polygon. The convex hull shape (CH-Shape) is defined according to the number of vertices of the $\mathrm{CH}$ polygon. For example, a convex hull with four vertices will create a rectangle. See Figure 2 for a detailed example.

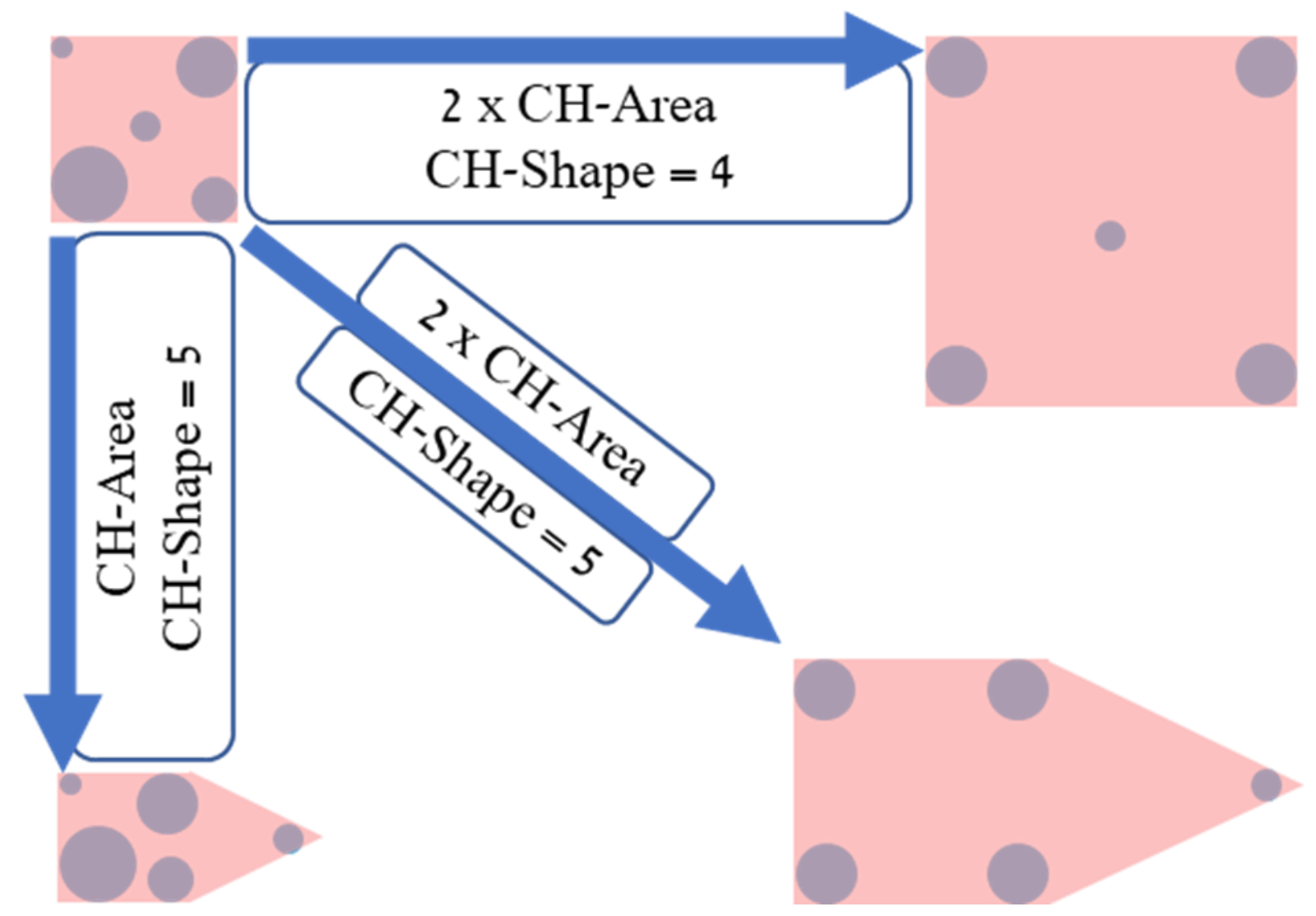

Fig. 2. The convex hull $(\mathrm{CH})$ has two main properties: $\mathrm{CH}$-Area and $\mathrm{CH}-\mathrm{Shape} \mathrm{CH}$-Area is defined according to the smallest contour creating a convex polygon containing all objects in the array. $\mathrm{CH}$-Shape is defined according to the number of vertices of the $\mathrm{CH}$ polygon. In the current example, the $\mathrm{CH}$-Shape and $\mathrm{CH}$-Area are individually manipulated. All arrays contain five items. The items in the right column have twice the $\mathrm{CH}$-Area as the items in the left column. Moreover, the bottom row items have a $\mathrm{CH}$-Shape with five vertices, while the top row items have four vertices. Therefore, each property of the $\mathrm{CH}$ (i.e., $\mathrm{CH}$-Area and $\mathrm{CH}-$ Shape) can be manipulated separately and thus they are distinct.

DeWind and colleagues (2015) presented an impressive model suggesting a dissociation between the ability to perform numerical judgements and the effect of physical properties. According to DeWind et al., non-symbolic numerical stimuli can be represented by 
a minimal set that includes total surface area, item surface area, $\mathrm{CH}$-Area, and sparsity (the average convex hull area per item). Importantly, DeWind's method uses a fixed item size or spacing and this may bound the number of possible $\mathrm{CH}$-Shapes. For example, imagine an array in the form of a triangle, comprised of three equally sized and evenly spaced dots. Adding one more equally sized and evenly spaced dot to the array will most probably create a quadrangle and not other $\mathrm{CH}$-Shapes. However, using dots in different sizes and spacing makes DeWind's model not suitable to represent $\mathrm{CH}-\mathrm{Shapes}$, because the number of $\mathrm{CH}$ vertices is independent from other properties of the array, as long as the item surface area and/or its spacing are not bounded (Hueter, 1994). Adding a dot in a different size and spacing to the array can create various $\mathrm{CH}-\mathrm{Shapes}$ : either a triangle or a quadrangle.

Salti et al.'s (2017) taxonomy points out that CH-Area is unique and a better candidate than other physical properties to underpin the processing of numerosity. This is true even a priori of analyzing how it affects processing because it represents the whole array using less information. Following the taxonomy, Salti and colleagues suggested a method to control the congruency effects of different properties while matching their saliency, regardless of their correlations with numerosity. They suggested choosing only one property at a time and carefully manipulating it, while the congruency of the rest of the physical properties would change in accordance with the changes in the chosen property. In a congruent trial, the ratio of one chosen property was equated to the numerical ratio, while all other physical properties were congruent to the numerical ratio, but not necessarily equal to it. In contrast, in an incongruent trial, one physical property was equal to the inverse of the numerical ratio, while the rest of the properties were also incongruent, but not necessarily equal to the inverse of the numerical ratio. Studies that used this method (Katzin et al., 2019; Salti et al., 2017) and other studies (Gebuis \& Reynvoet, 2012; Gilmore, Cragg, Hogan, \& Inglis, 2016) showed that the $\mathrm{CH}$-Area is the physical property that influences numerical comparisons. Importantly, these studies did not control for or attend the effects of $\mathrm{CH}$-Shape during numerical comparisons.

\subsection{Two counterparts of the convex hull}


The relationship between $\mathrm{CH}$-Area and $\mathrm{CH}-\mathrm{Shape}$ is unclear. It is unclear whether these two properties interact during numerical processing, and if so, how? Like the CH-Area, the $\mathrm{CH}$-Shape is an extrinsic property in the sense that it provides information on the whole array. The CH-Shape was also found to be predictive of numerosity (Buchta, 2006; Katzin et al., 2020). Moreover, at least for numerosities below 30, the number of $\mathrm{CH}$ vertices is more predictive of numerosity than CH-Area is (Cabo \& Groeneboom, 1994). Therefore, the CHShape is more probable to underlie numerical processing than the $\mathrm{CH}$-Area because it can be more useful in quantifying the number of items in an array. Figure 3 shows how the $\mathrm{CH}$-Shape probability distributions change as a function of numerosity. Katzin and colleagues (2020) found that participants' numerical estimations matched the $\mathrm{CH}-\mathrm{Shape}$ probability distributions. Accordingly, they suggested that participants rely on the natural probabilities of the $\mathrm{CH}$-Shape to estimate numerosity.

Classically, enumeration of more than four items is divided into the counting and the estimation ranges (between 5-10 items and above 10 items, respectively; e.g., Burr, Turi, \& Anobile, 2010; Trick \& Pylyshyn, 1993). In the counting range, if participants are provided with enough time, they will count the items in a serial, time-consuming and accurate manner. In the estimation range, the items are not counted but estimated. Likewise, $\mathrm{CH}$-Shape discriminability also changes as a function of the numerical range (see also Katzin et al., 2020). It is easier to discriminate between a dot array in the shape of a triangle and a dot array in the shape of a square than to discriminate between the shapes of arrays with 33 and 34 dots. Furthermore, as seen in Figure 3, the $\mathrm{CH}$-Shape distributions of different numerosities have different statistical properties, mainly different means, kurtosis, skewness, and variance. These differences are more pronounced when comparing numerosities in the counting range to numerosities in the estimation range. Thus, $\mathrm{CH}$-shape provides different amounts of information and discriminability for different numerical ranges. Consequently, the role of the $\mathrm{CH}-$ Shape during numerical processing might change as a function of the numerical range. Finally, the relationship between $\mathrm{CH}$-Area and $\mathrm{CH}$-Shape during numerical processing is not 
clear and might change as a function of the numerical range.
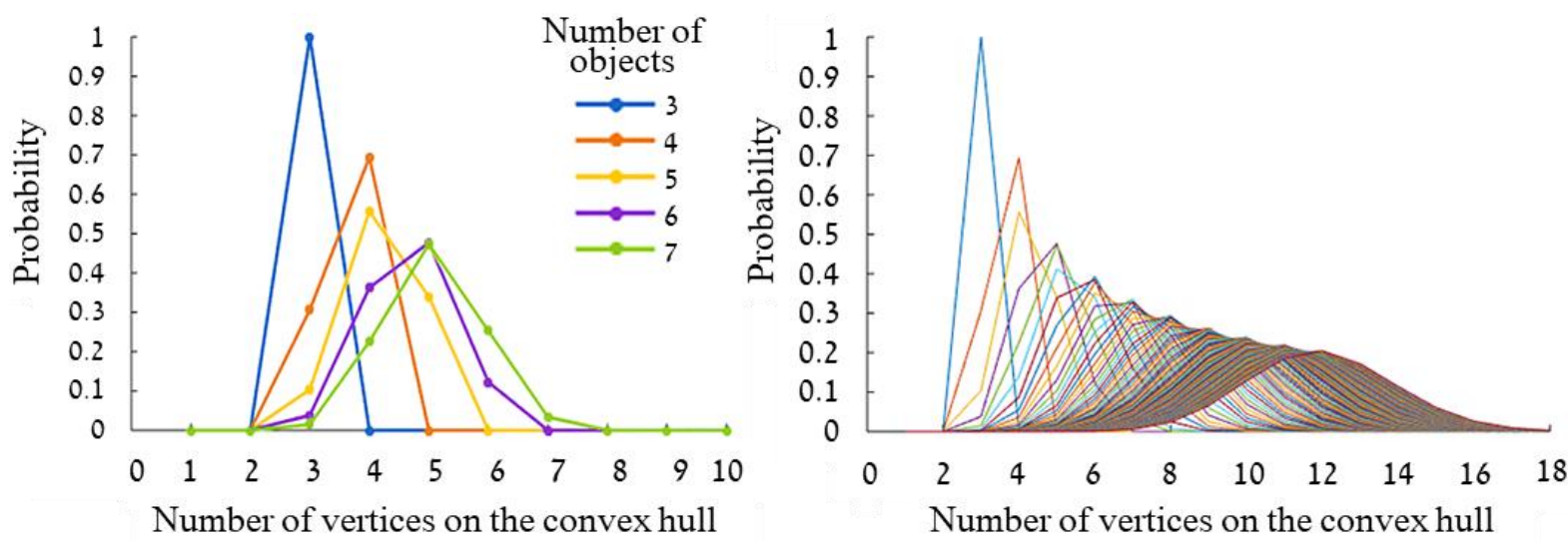

Fig. 3. The distribution of the CH-Shape (defined as the number of vertices on the $\mathrm{CH}$ polygon) for various numerosities. The left and right panels present the same curves only the right panel includes more numerosities. Each curve reflects a different number of dots. As the number of dots increases, the mean number of vertices increases (x-axis) and so does the probability of observing a higher number of vertices in a random array. Furthermore, as numerosity increases, so does the variance of the $\mathrm{CH}$-Shape probability distribution. This figure was created based on data collected by Katzin et al. (2020).

\section{The current study}

$\mathrm{CH}-\mathrm{Shape}$ and $\mathrm{CH}$-Area are both features of the same physical property - an array's convex hull. Accordingly, they can be used independently or together to compare the array's numerosities. The current research aimed to advance our understanding of the convex hull's effect during numerical comparisons, and the mechanism underlying this process. Our first objective was to examine whether $\mathrm{CH}$-Shape has a role in numerical comparisons in addition to its role in enumeration (Katzin et al., 2020). For that purpose, we re-analyzed the data from Katzin et al. (2019) and included data on the CH-Shape. We hypothesized that the CH-Shape would have an effect on performance above and beyond the effect of the rest of the stimuli physical properties, including the $\mathrm{CH}$-Area (that was specifically examined in Katzin et al.'s [2019] experiments). Our second objective was to understand the interaction between the CHShape and $\mathrm{CH}$-Area during numerical comparisons. We hypothesized that the effect of the $\mathrm{CH}$-Area would be modulated by the $\mathrm{CH}$-Shape. Moreover, the interaction between the $\mathrm{CH}$ - 
Area and the $\mathrm{CH}$-Shape would have the largest effect on performance when the $\mathrm{CH}$-Area was equated to numerosity. Our last objective was to test if the $\mathrm{CH}-\mathrm{Shape}$ effect changes as a function of numerosity. We hypothesized that the $\mathrm{CH}$-Shape would affect performance more when its number of vertices was in the counting range than in the estimation range.

\section{Method}

All the data were taken from Katzin et al. (2019). In their study, Katzin et al. did not record information on the $\mathrm{CH}$-Shape. We re-analyzed Katzin et al.'s dataset because we wanted to study the interference and facilitation of the $\mathrm{CH}$-Shape on processing of numerosity. A total of 1,440 images were re-analyzed (see Figure 4), and the total number of participants was 120 . The current method section provides the relevant details for the current re-analysis. Further details about the participants, procedure and stimuli — including the numerosities, numerical ratios and physical properties ratios — are detailed in the original method section of Katzin et al.'s study.

\subsection{Stimuli}

\subsubsection{Extracting data on $\mathrm{CH}$-Shape}

In the original experiment, information on the $\mathrm{CH}$-Shapes was not recorded. As such, we analyzed all stimuli used in the original experiment using a custom MATLAB R2019a code and recorded the number of vertices on the $\mathrm{CH}$ (see Figure 4). The original images were divided into two halves according to the line bisecting the image. Since all images were black/white they could be transformed into a binary matrix. Then the number of closed items was counted to account for the number of dots. Afterwards, the number of vertices on the convex hull was calculated. The code was uploaded and can be found online (see Supplementary A).

We sorted the data into three different congruency conditions according to $\mathrm{CH}$-Shape: Congruent-Shape (66.5\% of the trials) /Incongruent-Shape (25.5\% of the trials) /Same-Shape ( $8 \%$ of the trials). The difference between the conditions was based on the number of vertices. It could be either congruent or incongruent to numerosity, or equal in both arrays (i.e., Same- 
Shape). The original experiment was not designed to test the effects of $\mathrm{CH}$-Shape and therefore, $\mathrm{CH}-\mathrm{Shape}$ was not controlled in relation to numerosity. $\mathrm{CH}-\mathrm{Shape}$ ratio (mean $=0.8$, $S D=0.12$ ) was higher than numerosity ratio in most of the trials, and its congruency was not counterbalanced. We will expand on the possible implications of this design in the Discussion section.
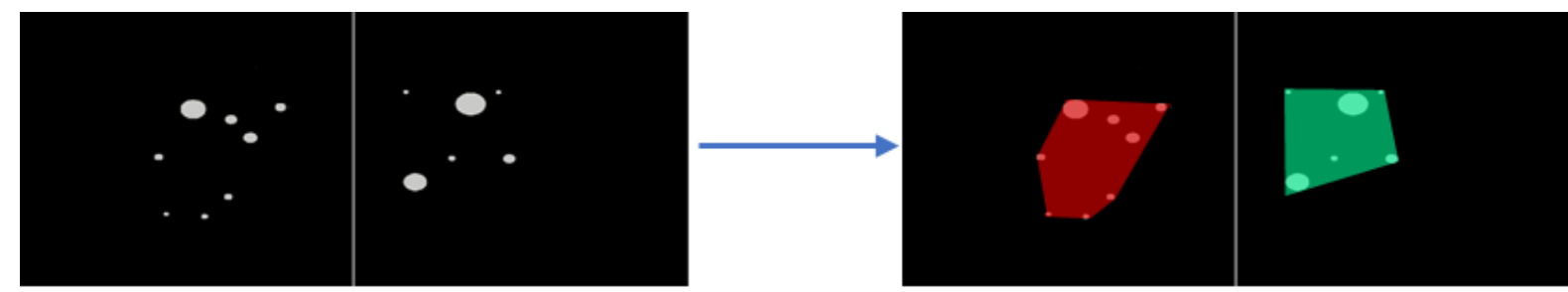

Fig. 4. Re-analysis of (unpublished) stimuli from Katzin et al. (2019). The stimuli from Katzin et al. were re-analyzed to record the number of vertices on the $\mathrm{CH}$, which was not recorded in the original experiment. The original images (left panel) were transformed into a binary matrix, after which the number of vertices on the convex hull was calculated (right panel).

\subsubsection{Analyzed stimuli}

Katzin et al.'s (2019) stimuli design was according to Salti et al.'s (2017) method. In the original experiment, five physical properties were manipulated: $\mathrm{CH}$-Area, average diameter, density, total circumference, and total surface area. There were three versions according to the Equated-Property. In each version, either the total surface area, average diameter, or the CHArea were equated (congruent) or inversed (incongruent) to numerosity. The congruency of the rest of the properties, except for the $\mathrm{CH}$-Shape, matched the congruency of the manipulated property. For example, when $\mathrm{CH}$-Area was controlled, in a congruent trial, the $\mathrm{CH}$-Area and numerosity ratios were equated. The rest of the properties were also congruent, such that they were bigger in the more numerous arrays of the stimuli, but their ratio was not necessarily equal to the numerical ratio. In an incongruent trial, the $\mathrm{CH}$-Area and numerosity ratios were inversed. The rest of the properties were also incongruent, such that they were bigger in the less numerous arrays of the stimuli, but their ratio was not necessarily equal to the inverse of the numerical ratio. The congruency of all physical properties, denoted as Other, except the $\mathrm{CH}-\mathrm{Shape}$, was counterbalanced, such that half of the trials were congruent, and the other half 
were incongruent. Throughout the text we will refer to the physical properties according to the congruency of the five physical properties that were manipulated (Other), or according to the three physical properties that could be equated to numerosity (Equated-Property), or according to the $\mathrm{CH}$-Shape congruency. The Numerical-Range was controlled such that the numerosities of half of the images (720) were within the counting range, the rest were within the estimation range.

\subsubsection{CH-Shape was not correlated with Other properties and was weakly correlated with numerosity}

We wanted to examine whether different physical properties were correlated with each other or with numerosity. Specifically, we inspected whether $\mathrm{CH}-\mathrm{Shape}$ could be predicted by the rest of the physical properties of the array $(\mathrm{CH}-\mathrm{Area}$, average diameter, density, total circumference, and total surface area). We found that $\mathrm{CH}$-Shape had relatively weak correlations with the rest of the physical properties with significant Pearson correlation coefficients ranging between $r=.074$ to $r=.105$ (see Figure 5). In contrast, the rest of the array's properties had higher correlations between them. Moreover, $\mathrm{CH}-\mathrm{Shape}$ and $\mathrm{CH}-\mathrm{Area}$ were not correlated, $r=.079$, as such, these are distinct properties. Finally, $\mathrm{CH}-\mathrm{Shape}$ had the lowest correlation with numerosity, $r=0.208$, in comparison to the correlation of the rest of the array's properties with numerosity, which ranged between $r=.267$ to $r=.586$. Figure 5 presents all the correlations including their significance levels and Bayesian evidence supporting them. 


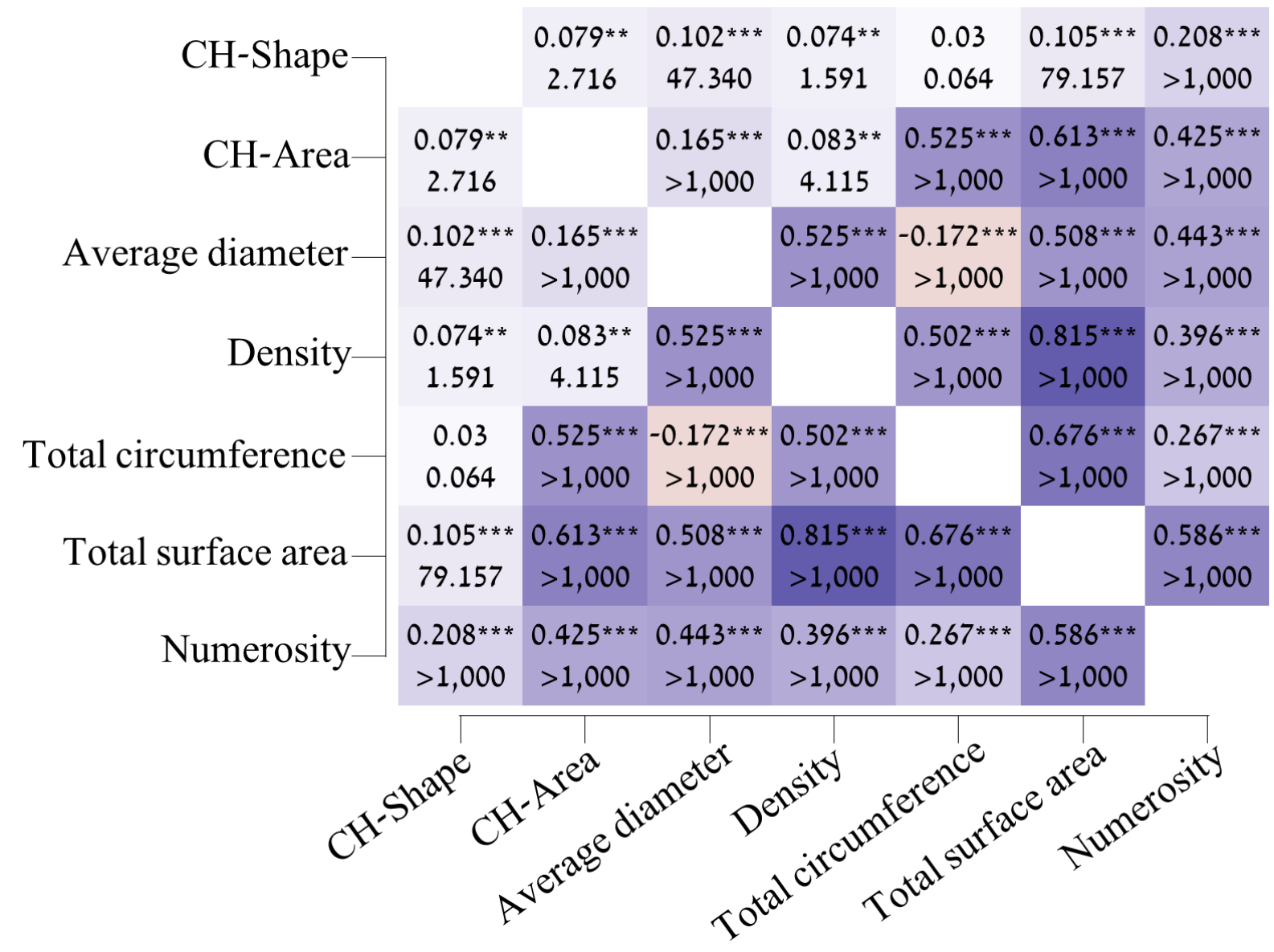

Fig. 5. Correlations between different physical properties of the arrays. The correlations between the different properties of Katzin et al.'s (2019) stimuli, including data on the CHShape, acquired through a re-analysis of the stimuli. Each cell in the matrix represents a different pairwise correlation, the top line depicts the Pearson correlation coefficient, and the bottom line presents $B F_{10}$ (Table 1 explains their interpretation). The purple colors represent positive correlations, while the pink colors represent negative correlations. The intensity of the color denotes the power of the correlation (i.e., darker shades represent stronger correlations). $N=1,440 ;{ }^{* *} p<.01,{ }^{* * *} p<.001$.

\subsection{Procedure and design}

Katzin et al. (2019) asked participants to perform two non-symbolic comparison tasks. Participants were either instructed to choose the more numerous array (Numerical-Task) or to choose the array that occupied more area (Physical-Task). We re-analyzed the Numerical-Task only, and excluded the Physical-Task, because we wanted to study the interference and 
facilitation of the $\mathrm{CH}$-Shape on processing of numerosity and not vice versa. Figure 6 presents a typical trial. Each trial began with a green fixation point presented for $500 \mathrm{~ms}$ on a black screen bisected by a vertical white line. The vertical bisecting line remained on screen throughout the trial. Afterwards, a blank black screen remained in view for 1,000 ms. Later, the stimulus comprised of two dots arrays appeared for $700 \mathrm{~ms}$. Participants could respond when viewing the stimulus or up to $1,100 \mathrm{~ms}$ after the stimulus disappeared and a blank screen was presented. In total, the maximal trial length was 2,500 ms. Participants were instructed to choose the more numerous array using the keyboard as fast and as accurately as they could. RT and accuracy rates were recorded. Participants were asked to press the "q" key if the left array was more numerous, and the "p" key if the right array was more numerous. Before the experiment started, there were six practice trials that included feedback. The Numerical-Task experiment included a total of 240 trials comprised of two blocks of 120 trials each.

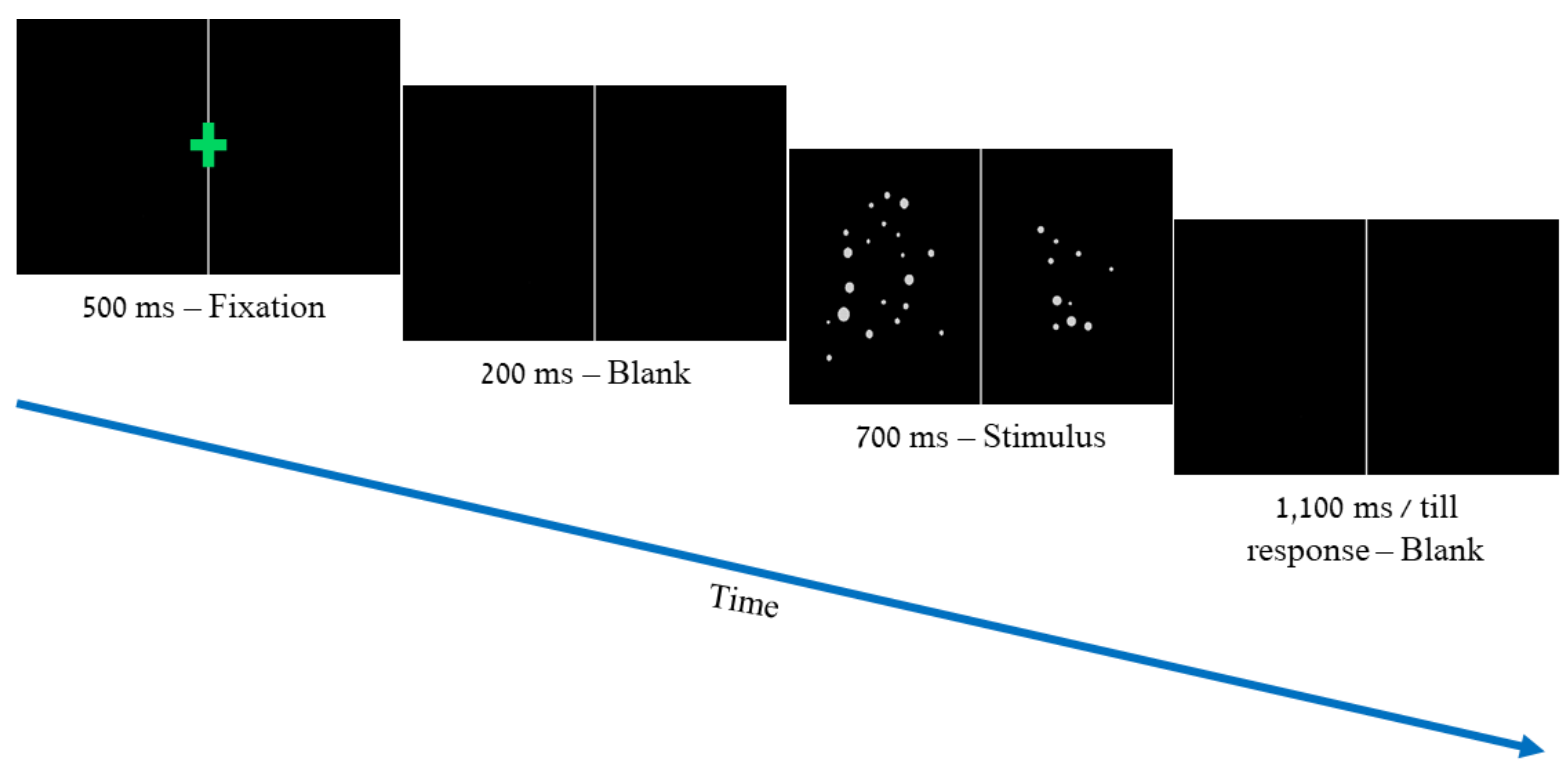

Fig. 6. A typical trial with a congruent stimulus. This is an example of a congruent stimulus with a numerical ratio of 10:20 for the dots. The design included a manipulation of congruency and of equating one of three physical properties (total surface area, the average diameter, or the $\mathrm{CH}$-Area). In the current example, the $\mathrm{CH}$-Area ratio was equated to the numerical ratio, and the $\mathrm{CH}$-Shape ratio of was also equal to that of numerosity with a ratio of $4: 8$ for the vertices on the convex hull. Since this is a congruent stimulus, the more numerous array was also higher in its Equated-Property ( $\mathrm{CH}$-Area), and the rest of its properties were also congruent to 
numerosity. Therefore, all five manipulated properties ( $\mathrm{CH}$-Area, average diameter, density, total circumference, and total surface area) were congruent to numerosity.

\section{Results and discussion}

\subsection{Pre-Processing}

For the RT analysis, we followed Katzin et al.'s (2019) analysis methodology and removed incorrect responses $(9.47 \%)$ and outliers (3.6\% of the remaining trials). Outliers were defined as trials in which the latency exceeded 2.5 standard deviations from the mean calculated per each subject and each condition. For the accuracy analysis, we removed only trials with extreme latencies of RT $<200$ or RT $>5,000 \mathrm{~ms}(0.03 \%)$.

\subsection{Analysis}

We analyzed how the $\mathrm{CH}$-Shape affected performance in a numerical comparison task. We also tested if the effect of the $\mathrm{CH}$-Area and the rest of the physical properties was modulated by the $\mathrm{CH}$-Shape. Moreover, we tested if the $\mathrm{CH}$-Shape effect changed according to one of the three properties that could be equated to numerosity. Finally, we analyzed whether the effect of the $\mathrm{CH}$-Shape changed between the counting and the estimation ranges.

A 4-way repeated measures analysis of variance (ANOVA) was used to analyze RTs and error rates. The between-subjects independent variables were the Equated-Property $\left(\mathrm{CH}^{-}\right.$ Area / total surface area / average diameter) and Numerical-Range (counting / estimation). The within-subject independent variables were grouped under the Other condition, defined as the congruency of all five physical properties besides the shape of the $\mathrm{CH}$ (Congruent-Other / Incongruent-Other), and CH-Shape (Congruent-Shape / Same-Shape / Incongruent-Shape). The $F$ statistic and the relevant degrees of freedom were corrected using the GreenhouseGeisser method (Abdi, 2010; Greenhouse \& Geisser, 1959). Pvalues were corrected using the Holm-Bonferroni correction (Holm, 1979). Finally, we performed a complementary Bayesian hypothesis testing analysis. The rational for conducting the Bayesian analysis was to provide an additional quantification to the standard $p$ values, and to perform null hypothesis testing (Keysers, Gazzola, \& Wagenmakers, 2020). We used JASP software to compute Bayes factors 
$(B F)$, using default JZS priors (Rouder, Morey, Speckman, \& Province, 2012; Rouder, Speckman, Sun, Morey, \& Iverson, 2009). To isolate the different interactions, we computed inclusion and Bayes factors (Clyde, Ghosh, \& Littman, 2011). The results of the inclusion Bayes factor method provide the change in posterior probability between models that include and models that exclude the relevant interaction. Inclusion Bayes factors ( $\left.B F_{\text {incl }}\right)$ were computed for the $\mathrm{H} 1$ testing. Exclusion Bayes factors ( $\left.B F_{\text {excl }}\right)$, calculated as the inverse of the Inclusion Bayes factors, were computed for the null hypothesis testing (Clyde et al., 2011). The interpretation of the Bayes factors was according to Jeffreys' scale (Jeffreys, 1998) and is presented in Table 1.

\section{Table 1}

Jeffreys' scale for Bayes factor interpretation

\begin{tabular}{cc}
\hline Bayes Factor & Evidence \\
\hline$<1$ & Anecdotal: not enough evidence \\
$1-3$ & Weak \\
$3-10$ & Moderate \\
$10-30$ & Strong \\
$30-100$ & Very strong \\
$>1,000$ & Decisive \\
\hline
\end{tabular}

Note: Jeffreys' scale (Jeffreys, 1998) is a reference frame for Bayes factors interpretation. The original scale was adapted to make the table easier to read, as the original table was presented using a scientific notation of powers of ten and more complicated definitions for the strength of evidence (Faulkenberry, Ly, \& Wagenmakers, 2020).

\subsubsection{RT analysis}

\subsubsection{CH-Shape affected RTS}

There was a significant main effect for $\mathrm{CH}$-Shape on RTs (see Figure 7$), F(1.6,185)=$ 31.385, $p<.001, \eta^{2} p=.216$. Decisive Bayesian evidence, $B F_{10}>1,000$, supported this effect. Therefore, changes in the CH-Shape affected participants' RTs. When the CH-Shape was incongruent, it hindered performance by $13 \mathrm{~ms}$ compared to when $\mathrm{CH}-\mathrm{Shape}$ was the same in both arrays, $t(119)=3.264, p<.001, d^{\prime}=.353$, with $B F_{10}=19.602$ reflecting strong evidence in 
favor of $\mathrm{H} 1$. Furthermore, when the $\mathrm{CH}-\mathrm{Shape}$ was congruent to numerosity, $\mathrm{RT}$ was faster by $13 \mathrm{~ms}$ than when it was the Same-Shape, $t(119)=4.051, p<.001, d^{\prime}=.370, B F_{10}=133.775$.

Importantly, there was decisive Bayesian evidence for the aforementioned simple effect.
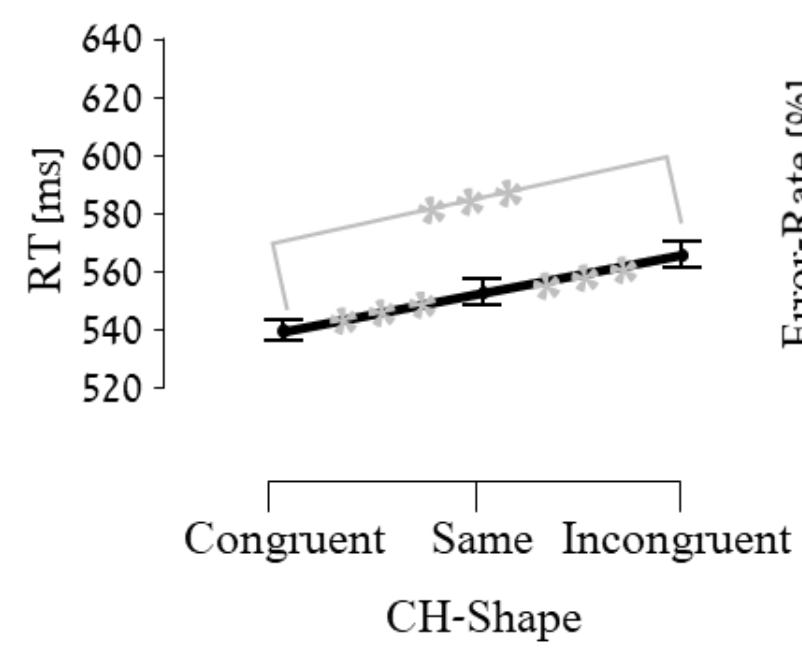
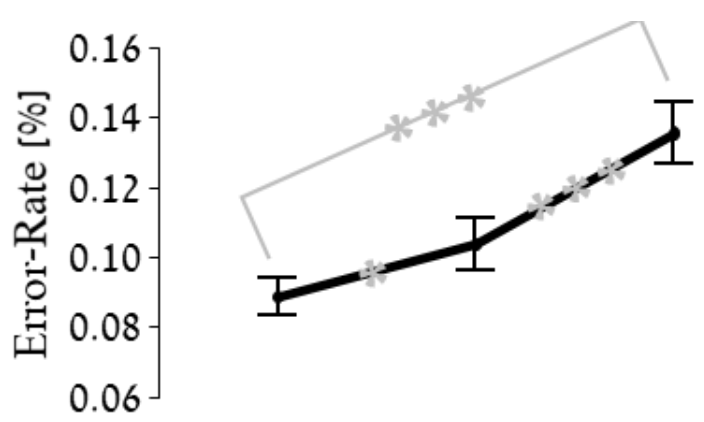

Congruent Same Incongruent CH-Shape

Fig. 7. RTs and Error-Rate as a function of CH-Shape. The left panel depicts RTs and the right panel depicts the error rates as a function of the $\mathrm{CH}$-Shape-Congruency condition. The $\mathrm{x}$-axis presents the different congruency condition: Congruent-Shape / Same-Shape / IncongruentShape. ${ }^{*} p<.05,{ }^{* * *} p<.001$.

\subsubsection{Other properties affected $R T$ s}

As expected following Katzin et al. (2019), there was a main RT effect for the congruency of the rest of the physical properties besides $\mathrm{CH}$-Shape (i.e., Other: $\mathrm{CH}$-Area, average diameter, density, total circumference, and total surface area), $F(1,114)=136.115, p<$ $.001, \eta^{2} \mathrm{p}=.544$. This effect was supported by decisive Bayesian evidence, $B F_{10}>1,000$, and was also demonstrated by others (e.g., Nys \& Content, 2011; Salti et al., 2017). Therefore, when physical properties were incongruent with numerosity, performance was hindered (see Figure 7).

\subsubsection{3. $C H$-Shape effect on $R T$ s interacts with Other properties}

There was a significant interaction between Other x CH-Shape (see Figure 8), F(1.7, 191) $=8.15, p<.001, \eta^{2} p=.067$, supported by a weak $B F_{\text {incl }}=2.193$. In general, when the $C H-$ 
Shape was incongruent, RTs were slower than when the $\mathrm{CH}$-Shape was congruent. However, this effect changed when the Other properties changed ( $\mathrm{CH}$-Area, average diameter, density, total circumference, and total surface area). When all Other properties were incongruent to numerosity, and $\mathrm{CH}$-Shapes were congruent, there was a facilitation of $23 \mathrm{~ms}$ in comparison to when $\mathrm{CH}-$ Shapes were the same in both arrays, $t(119)=5.207, p<.001, d^{\prime}=.176, B F_{10}>1,000$. In contrast, when all Other properties were congruent to numerosity, while $\mathrm{CH}-\mathrm{Shapes}$ were incongruent, there was an interference of $24 \mathrm{~ms}$ in comparison to when $\mathrm{CH}-\mathrm{Shapes}$ were the same in both arrays, $t(119)=5.333, p<.001, d^{\prime}=.184, B F_{10}>1,000$. This indicates that the effect of the $\mathrm{CH}$-Shape changed when congruency of the different physical properties changed, such that the interference caused by the $\mathrm{CH}$-Shape was modulated by the rest of the physical properties in a transient manner.

o Congruent-Other

- Incongruent-Other

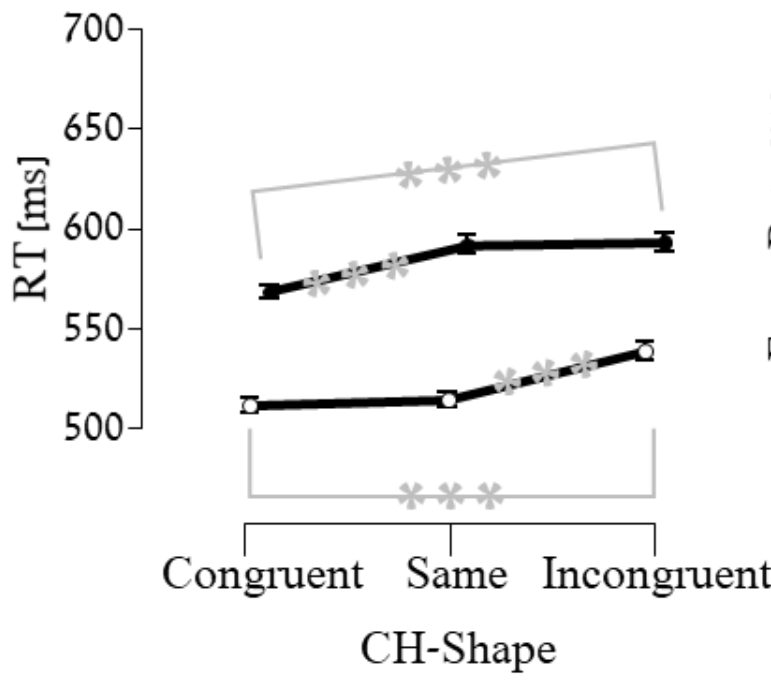

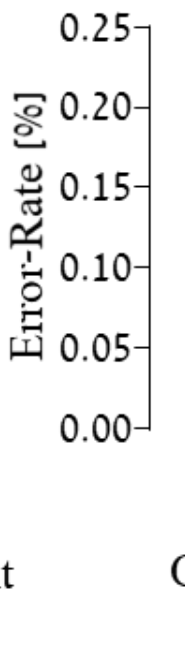

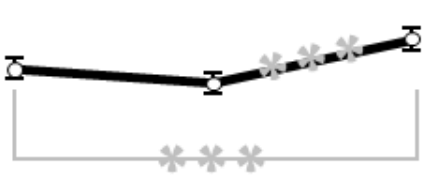

Congruent Same Incongruent $\mathrm{CH}-\mathrm{Shape}$

Fig. 8. RTs and Error-Rate as a function of $\mathrm{CH}$-Shape and Other properties congruency. RTs appear on the left panel and Error-Rate appears on the right panel. The x-axis presents the different CH-Shape congruency conditions: Congruent-Shape / Same-Shape / IncongruentShape. The white and black circles represent Other properties congruency conditions of Congruent-Other and Incongruent-Other (respectively). ${ }^{* *} p<.001$.

4.2.1.4. CH-Shape effect on RTs was persistent, regardless of the of the specific property that was equated to numerosity 
The interaction between Equated-Property $\mathrm{x}$ CH-Shape was not significant, $p=.075$, with strong evidence, $B F_{\text {excl }}=23.043$, supporting the null effect. Namely, the effect of the $\mathrm{CH}$ Shape was not modulated by the specific property that was equated to numerosity.

\subsubsection{CH-Shape affects $R$ Ts regardless of the Numerical-Range}

The interaction between Numerical-Range $\mathrm{x}$ CH-Shape was not significant, $p=.185$, with strong evidence supporting the null-hypothesis, $B F_{\text {excl }}=14.037$. Therefore, we suggest that $\mathrm{CH}$-Shape affected RTs, independent of the Numerical-Range (see Figure 9). An analysis of only the estimation range trials (see Supplementary B) confirmed that $\mathrm{CH}$-Shape influenced performance within the estimation range. This was intended to make sure that the CH-Shapes' effect on performance was not driven by the counting trials alone.

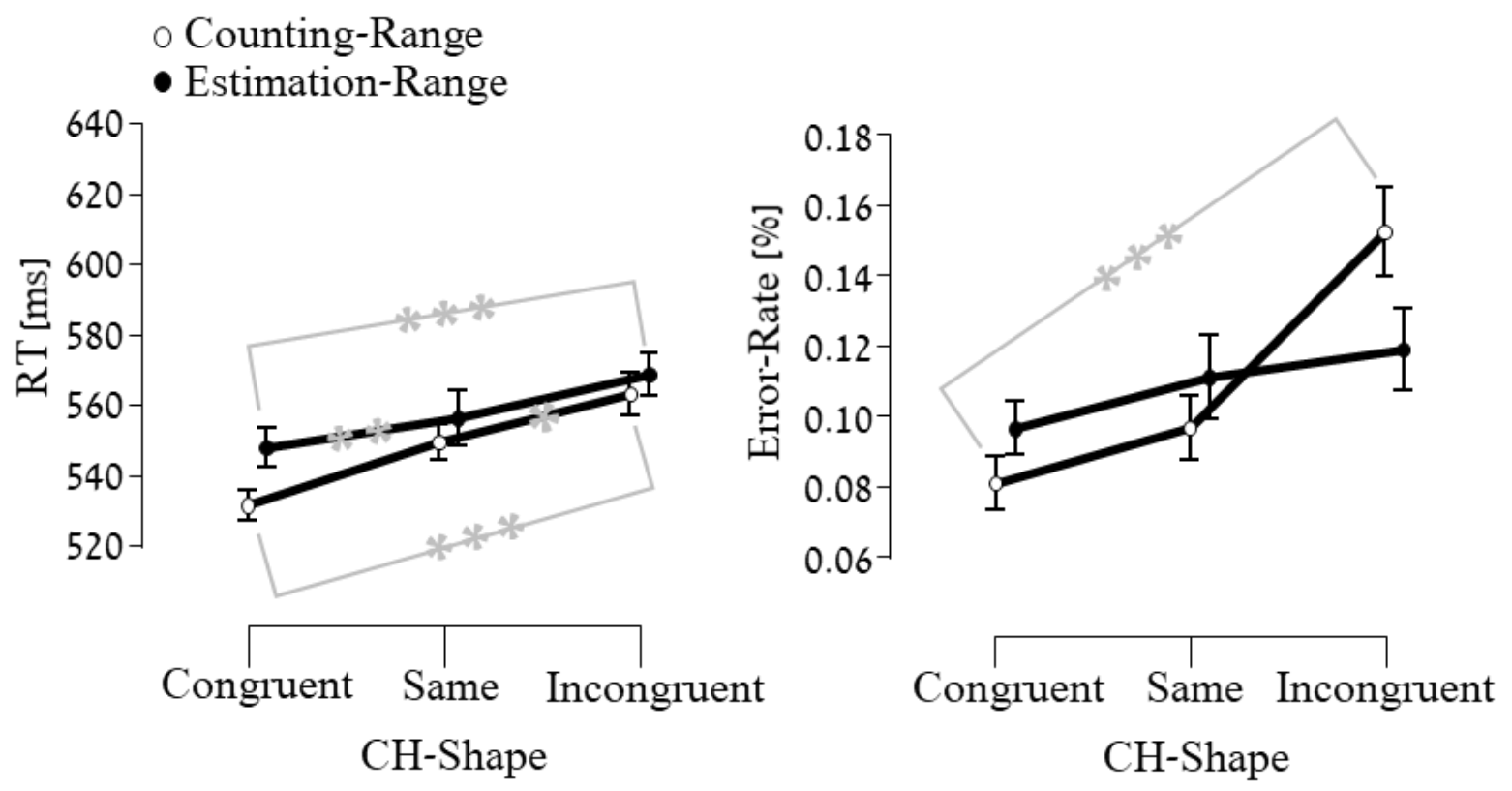

Fig. 9. RTs and Error-Rate interaction between Numerical-Range and CH-Shape. RTs and Error-Rate (left and right panels, respectively) in the counting and estimation ranges are displayed according to the $\mathrm{CH}$-Shape congruency condition. The $\mathrm{x}$-axis presents the different $\mathrm{CH}-\mathrm{Shape}$ congruency conditions: Congruent-Shape / Same-Shape / Incongruent-Shape. The white and black circles represent the different Numerical-Ranges of counting and estimation (respectively). ${ }^{*} p<.05,{ }^{* *} p<.01 .{ }^{* * *} p<.001$.

\subsubsection{Error-Rate analysis}




\subsubsection{CH-Shape affected Error-Rate}

There was a significant main effect for $\mathrm{CH}$-Shape on Error-Rate (see Figure 7), $F$ (2, 228) $=29.69, p<.001, \eta^{2} \mathrm{p}=.207$. Decisive Bayesian evidence, $B F_{10}>1,000$, supported this effect. As seen in Figure 7, Error-Rate increased by 4.7\% for Incongruent-Shapes in comparison to congruent $\mathrm{CH}-$ Shapes, $t(119)=7.559, p<.001, d^{\prime}=.69$, with decisive Bayesian evidence, $B F_{10}>1,000$. Also, Congruent-Shape trials had 1.5\% less errors than Same-Shape trials, $t(119)=2.481, p=.014, d^{\prime}=.227$, with moderate Bayesian evidence, $B F_{10}=8.255$. This indicated that changes in $\mathrm{CH}-\mathrm{Shape}$ could increase or decrease errors. Incongruent $\mathrm{CH}$-Shapes hindered performance, while congruent $\mathrm{CH}$-Shapes facilitated performance in comparison to the Same-Shape trials.

\subsubsection{Other properties affected Error-Rate}

As Katzin et al. (2019) found, there was a main effect for congruency of Other properties on Error-Rate, $F(1,114)=154.526, p<.001, \eta^{2} \mathrm{p}=.575$. This effect was supported by decisive Bayesian evidence of $B F_{10}>1,000$. Namely, when the stimuli properties, not including the $\mathrm{CH}$-Shape, were incongruent, it hindered performance (see Figure 8).

\subsubsection{CH-Shape effect on Error-Rate interacts with Other properties}

There was a significant interaction between Other $\mathrm{x} C H$-Shape, $F(2,228)=12.528, p<$ $.001, \eta^{2} \mathrm{p}=.099$, with decisive evidence supporting it, $B F_{\text {incl }}>1,000$. This indicated that the $\mathrm{CH}-$ Shape effect was modulated by Other properties (CH-Area, average diameter, density, total circumference, and total surface area). Namely, the $\mathrm{CH}$-Shape effect was more pronounced when the rest of the physical properties were incongruent to numerosity (see Figure 8). When Other properties were incongruent to numerosity, and $\mathrm{CH}$-Shapes were also incongruent then Error-Rate increased by $7.5 \%$ in comparison to Same-Shape trials, $t(119)=7.207, p<.001, d^{\prime}$ $=.25$, with a moderate $B F_{10}=3.325$. In contrast, Error-Rate decreased by $8 \%$ when Other properties referred to incongruent numerosity, while $\mathrm{CH}-\mathrm{Shapes}$ were congruent to numerosity in comparison to when the $\mathrm{CH}$-Shapes were the same in both arrays, $t(119)=$ 
$7.713, p<.001, d^{\prime}=.359$, with decisive $B F_{10}>1,000$. These results suggests that interference caused by the $\mathrm{CH}-\mathrm{Shape}$ was modulated by the rest of the physical properties in a transient manner.

4.2.2.4. CH-Shape effect on Error-Rate was persistent, regardless of the specific property that was equated to numerosity

The interaction between Equated-Property x CH-Shape was not significant, $p=.128$, with very strong evidence, $B F_{\text {excl }}=20.322$, supporting the null hypothesis. This suggests that the $\mathrm{CH}$-Shape effect did not change as a function of the specific property that was equated to numerosity (CH-Area, total-surface area, or average diameter).

4.2.2.5. CH-Shape affected Error-Rate in the counting and estimation ranges but the effect was more pronounced in the counting range

The interaction between Numerical-Range $\mathrm{x} C H$-Shape was significant, $F(2,228)=$ $10.072, p<.001, \eta^{2} \mathrm{p}=.081$. The interaction was also supported by strong evidence, $B F_{\text {incl }}=$ 27.718, in favor of $\mathrm{H} 1$. This result suggested that the $\mathrm{CH}$-Shape effect on Error-Rate was modulated by the Numerical-Range (see Figure 9). The effect of $\mathrm{CH}$-Shape was larger in the counting range, where Error-Rate was higher by $7 \%$ when $\mathrm{CH}-\mathrm{Shapes}$ were incongruent than when $\mathrm{CH}-$ Shapes were congruent to numerosity, $t(59)=7.391, p<.001, d^{\prime}=.954$, with decisive evidence, $B F_{10}>1,000$, supporting $H 1$. In contrast, in the estimation range the difference in Error-Rate between congruent CH-Shapes trials to incongruent CH-Shapes trials was not significant, $p=.215$, with moderate evidence, $B F_{01}=3.326$, supporting the null hypothesis. These results suggests that the $\mathrm{CH}$-Shape effect was more pronounced in the counting range than in the estimation range.

\section{General discussion}

In the current study we considered the influence of $\mathrm{CH}$-Shape on numerical comparisons. In the context of comparison tasks, the convex hull is usually considered only by its area. Importantly, it was shown mathematically (Buchta, 2006) that the CH-Shape is 
predictive of numerosity. Recently, it was shown that $\mathrm{CH}$-Shape affects enumeration (Katzin et al., 2020). We therefore were interested in examining the effect of $\mathrm{CH}$-Shape in comparison tasks, and the interaction of $\mathrm{CH}$-Shape with other physical properties and especially its counterpart, $\mathrm{CH}$-Area. Using image processing we re-examined the data collected by Katzin and colleagues (2019). Our re-examination showed that both $\mathrm{CH}$-Shape and rest of the physical properties of the array have distinct influence on numerical comparisons. Changes in $\mathrm{CH}-$ Shape affected both response latency and accuracy (see Figure 7). When compared to neutral stimuli comprised of the same $\mathrm{CH}$-Shapes, incongruent $\mathrm{CH}$-Shapes hindered performance, and congruent $\mathrm{CH}$-Shapes facilitated it. There was evidence that the effect of $\mathrm{CH}-\mathrm{Shape}$ was more pronounced in lower numerosities (see Figure 9): the effect of $\mathrm{CH}-\mathrm{Shape}$ was larger in the counting range compared to the estimation range. In addition, the effect of $\mathrm{CH}-\mathrm{Shape}$ was modulated by rest of the array's properties, regardless of the specific physical property that was equated to numerosity (see Figure 8). These effects appeared when $\mathrm{CH}$-Shape was completely irrelevant to the task at hand. Importantly, the $\mathrm{CH}-\mathrm{Shape}$ interference and facilitation resulting from the judgment of numerosity emerged even in an experimental design that was not designed for that purpose, which implies that the $\mathrm{CH}-\mathrm{Shape}$ has a robust role in numerical processing.

The current re-analysis unfolds the connection between the findings regarding the role of the $\mathrm{CH}$-Shape and other physical properties during numerical comparisons. Our results (see Figure 8) show that people perform numerical comparisons in an adaptive manner that changes according to the interplay between $\mathrm{CH}$-Shape and other physical properties (including $\mathrm{CH}$ Area). Discrepancies between $\mathrm{CH}$-Shape and the rest of the array's properties induce conflict and interfere with numerical comparisons. When the information provided by the $\mathrm{CH}-\mathrm{Shape}$ correlates with numerosity (Congruent-Shape), the effect of the rest of the properties changes. Specifically, when the CH-Shape is congruent it weakens the effects of incongruency of the rest of the array's properties and facilitate responses. This is additional evidence that numerical processing is not static but adaptive, and that numerical processing is sensitive to the physical properties of the stimulus and their relationship (e.g., Gilmore et al., 2016; Leibovich-Raveh, Stein, Henik, \& Salti, 2018; Salti et al., 2017). 
$\mathrm{CH}-$ Shape, or any other physical property for that matter, could have been more or less salient than other physical properties. Saliency differences between different physical properties might have pushed participants to rely on one property more or less than the others, regardless of their roles in non-symbolic numerical judgements. As suggested by Salti et al. (2017), it is possible to avoid this problem by equating the ratios of numerosity and one physical property at a time. Following Salti and colleagues, in the current experiment half of the trials were congruent and $\mathrm{CH}$-Area ratio (or total surface area, or average diameter ratios) and numerosity ratio were equal. The other half of the trials were incongruent, and $\mathrm{CH}$-Area ratio (or total surface area, or average diameter ratios) and numerosity ratio were exactly inversed. This allowed comparing the effects of different physical properties on numerosity comparisons, while ensuring these properties were dissociated from numerosity. However, in $92.5 \%$ of the trials, $\mathrm{CH}-$ Shape and numerosity ratios were not equal or exactly inversed, and therefore $\mathrm{CH}$-Shape was not completely dissociated from numerosity. Importantly, in the majority of the trials, $\mathrm{CH}-\mathrm{Shape}$ ratio (mean $=0.8, S D=0.12$ ) was higher than the ratio of $\mathrm{CH}$ Area (or total surface or average diameter ratios), which were equal to the numerical ratio (mean $=0.67, S D=.012$ ). Therefore, the discriminability (Fechner, 1948) of CH-Shape was lower than the rest of the physical properties that were manipulated, including $\mathrm{CH}$-Area. In other words, it was harder to discriminate between the arrays according to their shapes than according to the rest of the manipulated properties and yet, the $\mathrm{CH}-\mathrm{Shape}$ affected numerical comparisons. All in all, the current results highlight the importance of $\mathrm{CH}$-Shape: a property that has not received enough attention in the literature of numerical cognition.

We found that the $\mathrm{CH}$-Shape has larger effect in the counting range than in the estimation range (see Figure 9). The interaction between $\mathrm{CH}-\mathrm{Shape}$ and numerical range confirms our hypothesis that $\mathrm{CH}$-Shape affects performance in the counting range more than in the estimation range. This interaction might be related to two aspects of $\mathrm{CH}$-Shape: shape discriminability and predictive value. The ability to discriminate between shapes might modulate performance. As the number of vertices increases, it becomes harder to discriminate between them (Katzin et al., 2020). Consequently, it should be harder to use $\mathrm{CH}-\mathrm{Shape}$ as a whole within the estimation range than within the counting range. According to this line of 
thought, one of the reasons it is harder to dissociate between two CH-Shapes with 33 and 34 vertices than to dissociate between a triangle and a square is because the $\mathrm{CH}$-Shapes in the estimation range (e.g., with 33,34 . vertices) are less discriminable than a triangle and a square. Another possible reason for the change in $\mathrm{CH}$-Shape effect between the counting and estimation ranges is the amount of information provided by the $\mathrm{CH}-\mathrm{Shape}$. The $\mathrm{CH}-\mathrm{Shape}$ predicts numerosity, however it becomes less predictive as numerosity increases. The reduction in predictiveness reflects the fact that as the number of items increases so does the variance of their CH-Shape probability distribution (Buchta, 2006; Cabo \& Groeneboom, 1994; Katzin et al., 2020). Accordingly, the precision of numerical decisions that use the CH-Shape as a visual cue decreases above the estimation range. Various studies of perceptual learning have shown that people rely on the precision of information (Körding \& Wolpert, 2004; Suárez-Pinilla, Seth, \& Roseboom, 2018). Accordingly, people may change their use of CHShapes in numerical processing according to their predictive value. A recent model for numerosity perception suggests that the amount of information provided by each numerosity and the ability to make numerical judgements are inherently connected (Cheyette \& Piantadosi, 2020).

We hypothesized that interaction of the convex hull counterparts, $\mathrm{CH}$-Area and $\mathrm{CH}^{-}$ Shape, is unique. Specifically, we hypothesized that $\mathrm{CH}$-Area and $\mathrm{CH}-\mathrm{Shape}$ would have the largest effect on performance when the $\mathrm{CH}$-Area would be equated to numerosity. The current results give inconclusive evidence for such an effect. Even though the $\mathrm{CH}-\mathrm{Shape}$ effect was modulated by the $\mathrm{CH}$-Area, the current analysis indicated that this happens regardless of the specific property that was equated to numerosity (i.e., the $\mathrm{CH}$-Area, total surface area, and average diameter). Importantly, the design of the current study does not allow an exhaustive test for such a hypothesis. To fully understand the interplay between the $\mathrm{CH}$-Shape and $\mathrm{CH}$ Area, there is a need for a careful experimental manipulation in which the saliency of both will be controlled.

\section{Conclusions}


To conclude, we showed that a property of $\mathrm{CH}$, its shape, and the other physical properties of non-symbolic arrays, have unique contributions to numerical processing. Moreover, both modulate comparative numerical judgments when irrelevant to the task (i.e., comparing numerosities). Most of the work in numerical cognition has been devoted to $\mathrm{CH}$ Area whereas $\mathrm{CH}-\mathrm{Shape}$ has attracted less attention. We suggest that research should focus on $\mathrm{CH}$-Shape and its interplay with other physical properties that correlate with numerosity. 


\section{References}

Abdi, H. (2010). The Greenhouse-Geisser correction. In N. Salkind (Ed.), Encyclopedia of research design (p. 10). Thousand Oaks, CA: SAGE Publications.

Bentley, J. L., Kung, H. T., Schkolnick, M., \& Thompson, C. D. (1978). On the average number of maxima in a set of vectors and applications. Journal of the ACM (JACM), 25(4), 536543. https: //doi.org/10.1145/322092.322095

Buchta, C. (2006). The exact distribution of the number of vertices of a random convex chain. Mathematika, 53(2), 247-254. https: //doi.org/10.1112/S0025579300000127

Burr, D. C., Turi, M., \& Anobile, G. (2010). Subitizing but not estimation of numerosity requires attentional resources. Journal of Vision, 106), 20-20. https: //doi.org/10.1167/10.6.20

Cabo, A. J., \& Groeneboom, P. (1994). Limit theorems for functionals of convex hulls. Probability Theory and Related Fields, 100(1), 31-55. https: //doi.org/10.1007/BF01204952

Cheyette, S. J., \& Piantadosi, S. T. (2020). A unified account of numerosity perception. Nature Human Behaviour, 4, 1265-1272. https: //doi.org/10.1038/s41562-020-00946-0

Clyde, M., Ghosh, J., \& Littman, M. (2011). Bayesian adaptive sampling for variable selection and model averaging. Journal of Computational and Graphical Statistics, 20(1), 80-101. https: //doi.org/10.1198/jcgs.2010.09049

Day, A. M. (1988). Planar convex hull algorithms in theory and practice. Computer Graphics Forum, 73), 177-193. https: //doi.org/10.1111/j.1467-8659.1988.tb00608.x

DeWind, N. K., Adams, G. K., Platt, M., \& Brannon, E. (2015). Modeling the approximate number system to quantify the contribution of visual stimulus features. Cognition, 142, 247-265. https: //doi.org/10.1016/j.cognition.2015.05.016 
Efron, B. (1965). The convex hull of a random set of points. Biometrika, 52(3-4), 331-343. https: //doi.org/10.1093/biomet/52.3-4.331

Egner, T. (2007). Congruency sequence effects and cognitive control. Cognitive, Affective, \& Behavioral Neuroscience, 74), 380-390. https: //doi.org/10.3758/CABN.7.4.380

Faulkenberry, T. J., Ly, A., \& Wagenmakers, E.-J. (2020). Bayesian inference in numerical cognition: A tutorial using JASP. Journal of Numerical Cognition, 6(2), 231-259. https: //doi.org/10.5964/jnc.v6i2.288

Fechner, G. T. (1948). Elements of psychophysics, 1860. In W. Dennis (Ed.), Readings in the history of psychology. (pp. 206-213). Appleton-Century-Crofts. https: //doi.org/10.1037/11304-026

Gebuis, T., Cohen Kadosh, R., de Haan, E., \& Henik, A. (2009). Automatic quantity processing in 5-year olds and adults. Cognitive Process, 10(2), 133-142.

Gebuis, T., \& Reynvoet, B. (2011). Generating nonsymbolic number stimuli. Behavior Research Methods, 43(4), 981-986. https: //doi.org/10.3758/s13428-011-0097-5

Gebuis, T., \& Reynvoet, B. (2012). The interplay between nonsymbolic number and its continuous visual properties. Journal of Experimental Psychology: General, 141(4), 642-648. https: //doi.org/10.1037/a0026218

Gilmore, C., Cragg, L., Hogan, G., \& Inglis, M. (2016). Congruency effects in dot comparison tasks: Convex hull is more important than dot area. Journal of Cognitive Psychology, 28(8), 923-931. https: //doi.org/10.1080/20445911.2016.1221828

Greenhouse, S. W., \& Geisser, S. (1959). On methods in the analysis of profile data. Psychometrika, 24(2), 95-112. https: //doi.org/10.1007/BF02289823

Henik, A., \& Tzelgov, J. (1982). Is three greater than five: The relation between physical and semantic size in comparison tasks. Memory \& Cognition, 1044), 389-395. https: //doi.org/10.3758/BF03202431 
Holm, S. (1979). A simple sequentially rejective multiple test procedure. Scandinavian Journal of Statistics, 6(2), 65-70. http://www.jstor.org/stable/4615733.

Hueter, I. (1994). The convex hull of a normal sample. Advances in Applied Probability, 26(4), 855-875. doi: $10.2307 / 1427894$.

Jeffreys, H. (1998). Theory of probability (3rd ed). Clarendon Press; Oxford University Press.

Katzin, N., Katzin, D., Rosén, A., Henik, A., \& Salti, M. (2020). Putting the world in mind: The case of mental representation of quantity. Cognition, 195, 104088. https: //doi.org/10.1016/j.cognition.2019.104088

Katzin, N., Salti, M., \& Henik, A. (2019). Holistic processing of numerical arrays. Journal of Experimental Psychology: Learning, Memory, and Cognition, 45(6), 1014-1022. https: //doi.org/10.1037/xlm0000640

Keysers, C., Gazzola, V., \& Wagenmakers, E.-J. (2020). Using Bayes factor hypothesis testing in neuroscience to establish evidence of absence. Nature Neuroscience, 23(7), 788-799. https: //doi.org/10.1038/s41593-020-0660-4

Körding, K. P., \& Wolpert, D. M. (2004). Bayesian integration in sensorimotor learning. Nature, 4276971), 244-247. https: //doi.org/10.1038/nature02169

Leibovich, T., \& Henik, A. (2013). Magnitude processing in non-symbolic stimuli. Frontiers in Psychology, 4, 375. https: //doi.org/10.3389/fpsyg.2013.00375

Leibovich, T., Katzin, N., Harel, M., \& Henik, A. (2017). From "sense of number" to "sense of magnitude": The role of continuous magnitudes in numerical cognition. Behavioral and Brain Sciences, 40, e164. https: //doi.org/10.1017/S0140525X16000960

Leibovich-Raveh, T., Stein, I., Henik, A., \& Salti, M. (2018). Number and continuous magnitude processing depends on task goals and numerosity ratio. Journal of Cognition, 1(1), 19. https: //doi.org/10.5334/joc.22 
Liao, X. P., Lu, J., Ma, J. Y., Xue, J. H., \& Yu, X. M. (2012). Modeling of 2D layout design system based on convex hull plus rubber band analog. Applied Mechanics and Materials, 215-216, 1219-1223. https: //doi.org/10.4028/www.scientific.net/AMM.215216.1219

Nys, J., \& Content, A. (2011). Judgement of discrete and continuous quantity in adults: Number counts! The Quarterly Journal of Experimental Psychology, 65(4), 675-90. https: //www.tandfonline.com/doi/abs/10.1080/17470218.2011.619661

Piazza, M., Izard, V., Pinel, P., Bihan, D. L., \& Dehaene, S. (2004). Tuning curves for approximate numerosity in the human intraparietal sulcus. Neuron, 44(3), 547-555. https: //doi.org/10.1016/j.neuron.2004.10.014

Rouder, J. N., Morey, R. D., Speckman, P. L., \& Province, J. M. (2012). Default Bayes factors for ANOVA designs. Journal of Mathematical Psychology, 56(5), 356-374. https: //doi.org/10.1016/j.jmp.2012.08.001

Rouder, J. N., Speckman, P. L., Sun, D., Morey, R. D., \& Iverson, G. (2009). Bayesian t tests for accepting and rejecting the null hypothesis. Psychonomic Bulletin \& Review, 16(2), 225-237. https://doi.org/10.3758/PBR.16.2.225

Rousselle, L., Palmers, E., \& Noël, M.-P. (2004). Magnitude comparison in preschoolers: What counts? Influence of perceptual variables. Journal of Experimental Child Psychology, 871 ), 57-84. https: //doi.org/10.1016/j.jecp.2003.10.005

Salti, M., Katzin, N., Katzin, D., Leibovich, T., \& Henik, A. (2017). One tamed at a time: A new approach for controlling continuous magnitudes in numerical comparison tasks. Behavior Research Methods, 49(3), 1120-1127. https: //doi.org/10.3758/s13428-016$0772-7$

Soltész, F., Szúcs, D., \& Szűcs, L. (2010). Relationships between magnitude representation, counting and memory in 4- to 7-year-old children: A developmental study. Behavioral and Brain Functions, 6(1), 13. https: //doi.org/10.1186/1744-9081-6-13 
Suárez-Pinilla, M., Seth, A. K., \& Roseboom, W. (2018). Serial dependence in the perception of visual variance. Journal of Vision, 18(7), 4. https: //doi.org/10.1167/18.7.4

Trick, L. M., \& Pylyshyn, Z. W. (1993). What enumeration studies can show us about spatial attention: Evidence for limited capacity preattentive processing. Journal of Experimental Psychology: Human Perception and Performance, 19(2), 331-351. https: //doi.org/10.1037//0096-1523.19.2.331

Weisstein, E. W. (2002). Convex polygon. From MathWorld-A Wolfram Web esource. https: //mathworld.wolfram.com/ConvexPolygon.html 